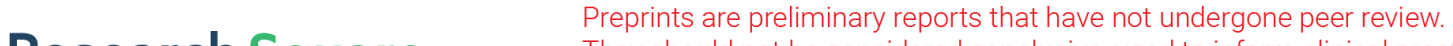 \\ They should not be considered conclusive, used to inform clinical practice, or referenced by the media as validated information. \\ The architecture and structure of alpaca skin (Vicugna pacos): impacts on industry and wool trade
}

Tais Harumi de Castro Sasahara ( $\sim$ tsasahara@usp.br)

Universidade de Sao Paulo https://orcid.org/0000-0002-4871-5625

\section{Concepta Margaret McManus}

Universidade de Brasilia Instituto de Ciencias Biologicas

Luciana Milagros Durand Neira

Universidade de Sao Paulo

Jessica Borghesi

Universidade de Sao Paulo

Marcella Kato

Universidade de Sao Paulo

Alice Del Colletto

Universidade de Sao Paulo

Ana Claudia Oliveira Carreira

Universidade de Sao Paulo

Silvio Pires Gomes

Universidade de Sao Paulo

Guilherme Chiarello

Universidade de Sao Paulo

Maria Angelica Miglino

Universidade de Sao Paulo

\section{Research}

Keywords: Histology, Stereology, Scanning electron microscopy, immunohistochemistry

Posted Date: May 12th, 2020

DOI: https://doi.org/10.21203/rs.3.rs-26867/v1

License: (a) This work is licensed under a Creative Commons Attribution 4.0 International License.

Read Full License 


\title{
The architecture and structure of alpaca skin (Vicugna pacos): impacts on industry and wool trade
}

Tais Harumi de Castro Sasahara1, Concepta Margaret McManus2, Luciana Milagros Durand Neira1, Jessica Borghesi1, Marcella Kato1, Alice Del Colletto1, Ana Claudia Oliveira Carreira1, Silvio Pires Gomes1, Guilherme Chiarello1, Maria Angelica Miglino1*

1. Department of Surgery, School of Veterinary Medicine and Animal Science, University of São Paulo, São Paulo, Brazil

2. Instituto de Ciências Biológicas, Campus Darcy Ribeiro, Universidade de Brasília, Brasília, DF, 79910-900

* Corresponding author:miglino@usp.br

\begin{abstract}
Background: Alpacas are reared mainly for fiber extraction, which is a highly valued product in the textile industry. For this reason, this work aims to evaluate the morphological and quantitative aspects of the light and dark alpaca skin of Huacaya and Suri alpacas, comparing the structure and architecture of the scapular, costal and lateral femoral skin. Biopsies were collected from the skin of 12 alpacas from the Pacomarca Experimental Fund, located at Puno - Peru. The samples were weighed and fixed in 10\% aqueous formalin solution for histological procedures. The histological sections were stained with Hematoxylin eosin, Picrossirius red and Masson Trichromic and immunostained for types I, III and IV collagen and S100.
\end{abstract}


Results: The derma presented sebaceous and sweat glands, as well as follicular groups with primary and secondary hair follicles. Each follicle had a hair called fiber, some with medulla and some without, but both surrounded by cortex and cuticle. The skin presented similar immunostaining for type I, II and IV and S100. Collagen III was detected only in the derma. The total volume of the skin, derma, follicular groups and sebaceous and sweat glands was estimated by stereology for the three body regions for both Huacaya and Suri alpacas. The total volume of skin (Vref) and total volume of follicular groups (VGF) were different for body regions. Femoral region showed higher values for VGF. Colour and breed were also different for Vref and total volume of derma (VD).

Conclusion: Comparing the two breeds the femoral region presented higher fiber production. Dark animals had more derma and it was reported close relationship between total skin volume and their fractions volumes: derma, follicular groups and sweat glands. Keywords: Histology, Stereology, Scanning electron microscopy, immunohistochemistry.

\section{Background}

Alpacas (Vicugna pacos) are a group of South American camelids, found in the upper Andean regions of South America. There are two varieties: the Huacaya and Suri. Huacaya represents $85 \%$ of the total alpacas in Peru and is characterized by its abundant, long and curly fiber, which has a spongy appearance. Suri is characterized by its smooth, slightly wavy, silky fiber. Alpaca fleece is one of the most valuable animal products in the textile industry and has a wide range of colors, almost 23 shades of white, beige, brown, gray and black $[1,2]$. Alpaca fiber diameter is important because it determines the commercialization price, which is complemented with the weight of the fleece [3-10].

The dermis is composed of collagen and elastic fibers, being responsible for the resistance and elasticity and this is where the blood vessels, lymphatics, nerves, hair 
follicles, sebaceous and sweat glands are located [11]. There is a negative correlation between follicular density and fiber thickness. The main histological characteristic of thin fibers is the larger number of secondary follicles, as it has been widely demonstrated that animals with smaller fiber thickness have a higher number of secondary follicles [12-14].

The fleece varies according to the body region, with the thinnest, longest and more dense in the dorsal, costal, sacral, brachial and femoral subregions. It is known that $80 \%$ of the fleece is made of delicate fibers, and $20 \%$ of the thickest fleece is on the head, as well as in the carpal and tarsal regions [15-17].

For these reasons, the integument of three body regions of the two varieties of alpacas (Huacaya and Suri) was evaluated to establish comparisons between them and to define which of these regions would produce the best fiber for commercial purposes.

\section{Methods}

Alpaca skin biopsies were collected from the Pacomarca Experimental Fund in Llalli, Melgar, Puno - Peru, from Inca Tops SA. Twelve alpacas of both sexes, aged 5 to 19 months, were used (Six Huacaya variety and six Suri variety alpacas, divided into two groups for light and dark tones respectively). Skin biopsies were performed with an $8 \mathrm{~mm}$ punch in three body regions: scapular, costal and lateral femoral and fixed in $10 \%$ formaldehyde. They were later cut into $2 \mathrm{~mm}$ thick fragments. Of the total fragments generated, $1 / 4$ was destined for scanning electron microscopy, $1 / 4$ for microscopic analysis, $1 / 4$ for immunohistochemical analysis and $1 / 4$ for stereological analysis. The distribution of fragments in these four sets was made following the pattern of systematic uniform random sampling (SURS). 


\section{Scanning electron microscopy}

The alpaca skin fragments were washed with distilled water and then additional washes were made in ultrasonic distilled water. The fragments were dehydrated in increasing concentrations of alcohols $(70 \%, 80 \%$ and $90 \%)$. The samples were then dehydrated in a critical point Leica EM CPD300 and placed on an aluminum disc (stub) to be gold plated in the Emitech K550 metallizer. Samples were analyzed using the LEO 435VP Scanning Electron Microscope (SEM) (Zeiss, Germany) from the Advanced Center for Diagnostic Imaging (CADI).

\section{Light microscopy}

Biopsies were dehydrated in an increasing series of ethanol concentrations $(70 \%, 80 \%, 90 \%$ and $100 \%)$ followed by xylene. The fragments were then embedded in paraffin as described by [18]. Longitudinal and transverse skin sections were cut in an automatic microtome (Leica, RM2165, Germany). The sections were stained with Hematoxylin and Eosin (HE), Masson's Trichrome and Picrosirius Red. The slides were analyzed under a Fluorescence Light Microscope (FLM) (Nikon Eclipse 80i, Japan). Those slides stained with Picrosirius Red were also observed under polarized light and images were acquired with AxioCam HRc camera (Zeiss, Germany) connected to an Olympus BX60 Microscope (Olympus, Japan).

\section{Immunohistochemistry}

The EnVision ${ }^{\mathrm{TM}}$ Flex kit, High pH, (Link), a high-sensitivity visualization kit used together with Autostainer Link (code K8000, Agilent Dako, USA), was used. Tissue sections were deparaffinized and hydrated. The slides were submerged in Flex Target Retrieval Solution (50x) previously heated to $95^{\circ} \mathrm{C}$ in a water bath for 20 minutes, followed by cooling at room temperature for 20 minutes. The slides were washed twice for 5 minutes with Flex Wash Buffer (Agilent Dako, USA) (20x). Peroxidase-Blocking 
Reagent was added for 30 minutes at room temperature. Samples were washed twice with Flex Wash Buffer (Agilent Dako, USA), 5 minutes each. The sections were then incubated in a humid chamber overnight at $4^{\circ} \mathrm{C}$, with the primary antibody (Collagen I clone (5D8-G9 / Col 1), GTX 60939, rabbit GeneTex brand polyclonal, at a 1:50 dilution; Collagen III (1E7-D7 / Col 3) GTX 60940, GeneTex mouse monoclonal mouse 1:50 dilution; LSBio (Life Span BioSciences Inc.) Collagen IV LS-B8763 rabbit polyclonal (IgG) at a 1: 100 dilution; S100A4 LS-B11817 rabbit polyclonal LSBio (Life Span BioSciences, Inc) at a 1: 100 dilution. For the negative control, the sections were incubated with phosphate buffered solution (PBS).

\section{Stereology}

\section{Total volume of the skin (reference volume)}

The $2 \mathrm{~mm}$-fragments, sampled previously, were embedded in paraffin and $5 \mu \mathrm{m}$ sections were collected on slides and stained with Masson trichrome. The distance between sections was $100 \mu \mathrm{m}$. The total volume of the skin (Vref) was estimated using the Cavalieri Method $[19,20]$. The image sections were captured using a digital camera connected to the light microscope (Nikon Eclipse 80i, Japan). The following formula was applied to estimate the total volume of skin (Vref):

$\boldsymbol{V}_{\text {ref }}=\sum p S \times a(p) \times t \times k$

$\sum p S$ : points hitting the whole skin fragment; $\mathrm{a}(\mathrm{p})$ : area of points (square distance between points), t: section thickness; $\mathrm{k}$ : the distance between the sections.

\section{Tissue shrinkage}

The 8mm-biopsy-fragments were weighed using a digital precision balance (model JK3202B) and their wet weights were converted into volumes considering a tissue density of $1.06 \mathrm{~g} / \mathrm{cm} 3$. After the estimation of the total volume of the skin by the Cavalieri method above, the tissue shrinkage was calculated as $15 \%$. 


\section{Total volume of the derma, follicular groups, sebaceous and sweat glands}

The total volume of the derma (VD), follicular groups (VGF), sebaceous (VGSe) and sweat glands (VGSu) and the fraction volume were calculated applying the following formulae:

$$
\begin{aligned}
& >V v D=\sum p D / \sum p P \\
& >V v G F=\sum p G F / \sum p P \\
& >V v G S e=\sum p G S e / \sum p P \\
& >V v G S u=\sum p G S u / \sum p P
\end{aligned}
$$

$\left(\sum \mathrm{pD}\right)$ : total points hitting the derma

( $\left.\sum \mathrm{pGF}\right)$ : total points hitting the follicular groups

( $\sum$ pGSe): total points hitting the sebaceous glands

( $\left.\sum \mathrm{pGSu}\right)$ : total points hitting the sweat glands

$\left(\sum \mathrm{pP}\right)$ : total points hitting the skin

The total volume was estimated multiplying each fraction volume by the reference volume. A three-way analysis of variance was carried out including factors of Breed, colour, region of the body and their interactions. Significant differences were tested using the tukey test at 5\%. Correlations and principal component analyses between characteristics were calculated. Analysis were carried out in SAS v9.4 (Cary, North Carolina).

\section{Results}

\section{Scanning electron microscopy}

The two constituent layers of the skin were visualized: the epidermis and the dermis. In addition, there was the presence of adipose tissue-associated hypodermis (Fig. 1a). In the dermis, it was possible to observe the hair bulbs and the follicular groups, 
which determine the grouping of hair follicles, surrounded by the connective tissue of the dermis (Fig. 1b and Fig. 1c).

The fiber was observed emerging from the epidermis, with the presence of the outermost layer, the cuticle, arranged in scales (Fig. 1d). At the junction between the epidermis and the dermis, it was possible to observe a cluster of cells irregularly arranged in the epidermis, called the basal layer. Still in the epidermis, the stratum corneum was seen, which is the last layer (Fig. 1d and Fig. 1e). In the papillary dermis, there was a group of six fibers emerging from the same hair follicle (Fig. 1f). Adjacent to the hair follicles were the sebaceous glands (Fig. 1g).

\section{Histology}

In transverse sections, it was seen that alpaca skin is composed of the epidermis and dermis. It was also possible to visualize the hypodermis or subcutaneous tissue as shown in Figures $2 \mathrm{a}$ and $2 \mathrm{~b}$. The epidermis and dermis were separated by the basal or germ layer of the epidermis (Fig. 2c and Fig. 2d).

The dermis was located between the epidermis and the hypodermis and consisted of the sweat glands as shown in Fig. 2e. The sebaceous gland consisted of rounded cells and acidophilic nuclei, which were arranged near a hair follicle. The dermis contained follicular groups, structured by a set of hair follicles grouped into a species of nests (Figs. $2 \mathrm{e}-2 \mathrm{~g})$. In the dermis, the sebaceous gland was visible, that is a tubular gland located inside a follicular group (Fig. $2 \mathrm{~g}$ ). The hair follicles usually contained more than one hair shaft or fiber inside. They were called composite follicles, which are seen in Fig. 6h, where it was possible to verify the presence of four fibers without marrow in a single hair follicle. Primary follicles consisted of three structures: the medulla internally, the cortex and the cuticle surrounding the hair cortex. Three layers of the hair follicle were observed: the inner sheath, consisting of a cuticle, a granular epithelial layer (consisting of flattened 
cells), and a pale epithelial layer (outer layer of cuboid cells), followed by the outer sheath. The last layer was the vitreous membrane (Fig. 2i).

In the reticular dermis, hair bulbs surrounded by dense connective tissue were observed, which did not emerge evenly (Fig. $2 \mathrm{j}$ and Fig. $2 \mathrm{k}$ ). The hair bulb contained the hair papilla or dermal papilla. The cells of the central portion of the matrix formed the hair medulla and, surrounding the dermal papilla, melanocytes within the hair cortex were identified (Fig. 21). In the longitudinal sections, the dermis contained collagen fibers, compared to the epidermis which did not present this constitution. No collagen was found highlighted in the blue color of the dermis (Figs. 3a-3c).

The hair follicles were arranged into follicular groups located in the dermis and surrounded by collagen fibers as shown in Figs. 3d-3f. The hair follicles, found in the deep dermis, had a higher quantity of blue-stained collagen. Longitudinal sections of the skin were made, and it was observed that, in the dermis, the follicular groups were surrounded by a larger quantity of collagen (Figs. 3i-31).

From the Picrosirius Red staining performed on the transverse histological sections of the skin, the dermis and hypodermis showed collagen fibers (Figs. 4a-4c). These were confirmed by the polarization technique, again showing the collagen fibers dispersed by the dermal tissue (Fig. $4 \mathrm{~d}$ and Fig. 4e). This technique allowed the differentiation of collagen fiber types, with red and orange fibers compatible with type I collagen fibers interspersed with green fibers, indicating reticular fibers or type III collagen fibers (Fig. 4f).

In both Picrosirius Red staining and polarized light analysis, follicular groups delimited by collagen fibers were observed. However, in the hair follicles only collagen fibers are observed in the outer layer. Most collagen fibers were red to orange and therefore considered collagen type I fibers (Figs. 4g-4l). 


\section{Immunohistochemistry}

In the four groups of alpacas: light-fiber Huacaya (Fig. 5 a, e, i, n, r), dark-fiber Huacaya (Fig. 5 b, f, j, o, s), light-fiber Suri (Fig. 5 c, g, 1, p, t) and dark-fiber Suri (Fig. $5 \mathrm{~d}, \mathrm{~h}, \mathrm{~m}, \mathrm{q}, \mathrm{u})$, when antibodies for Collagen Type I, Collagen Type IV and S100 were used, the structures of the epidermis, some collagen fibers of the dermis, hair follicles, sebaceous glands and sweat glands were marked, which was observed for the scapular, costal and lateral femoral regions.

\section{Quantification}

Vref and VGF were significantly different for body region (Table 1), with femoral region showing higher values than scapula, but costal was not different from femoral or scapula for VGF (Table 2). There were also significant interactions between colour and breed for Vref and VD. Dark coats had significantly lower values in Huacaya than Suri breeds for both traits while VD was lower in light than dark coats within the Suri breed (Table 3).

Correlations between Vref and VD were high and medium with VGSu, while others were low (Table 4).

Table 1. Level of Significance and summary of analysis of variance for coat traits in alpacas

\begin{tabular}{|l|c|c|c|c|c|}
\hline & Vref & VGF & VD & VGSe & VGSu \\
\hline Source & Pr $>$ F & Pr $>$ F & Pr $>$ F & Pr > F & Pr $>$ F \\
\hline Breed & 0.00 & 0.09 & $<.0001$ & 0.32 & 0.15 \\
\hline Colour & 0.52 & 0.60 & 0.12 & 0.24 & 0.91 \\
\hline Breed*Colour & 0.05 & 0.62 & 0.00 & 0.29 & 0.75 \\
\hline Region & 0.02 & 0.00 & 0.30 & 0.48 & 0.38 \\
\hline Breed*Region & 0.40 & 0.24 & 0.70 & 0.31 & 0.93 \\
\hline Colour*Region & 0.53 & 0.38 & 0.89 & 0.52 & 0.75 \\
\hline Breed*Colour*Region & 0.23 & 0.37 & 0.47 & 0.32 & 0.52 \\
\hline
\end{tabular}




\begin{tabular}{|l|c|c|c|c|c|}
\hline $\mathrm{R}_{2}$ & 0.58 & 0.52 & 0.66 & 0.32 & 0.21 \\
\hline $\mathrm{cv}$ & 19.34 & 21.09 & 30.06 & 177.83 & 47.11 \\
\hline Mean mm3 & $4.6 * 109$ & $2.3 * 109$ & $1.6 * 109$ & $5.5 * 107$ & $1.7 * 108$ \\
\hline
\end{tabular}

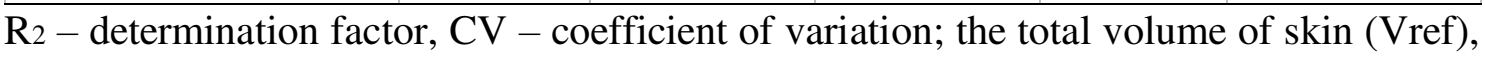
total volume of the derma (VD), follicular groups (VGF), sebaceous (VGSe) and sweat glands (VGSu)

Table 2. Means by Region for total volume of skin (Vref) and follicular groups (VGF) in alpacas in $\mathrm{mm} 3$

\begin{tabular}{lc|c|}
\hline Region & Vref & VGF \\
\hline Femoral & $5.2 * 109 \mathrm{a}$ & $2.8 * 109 \mathrm{a}$ \\
\hline Costal & $4.4 * 109 \mathrm{ab}$ & $2.0 * 109 \mathrm{~b}$ \\
\hline Scapula & $4.2 * 109 \quad \mathrm{~b}$ & $2.2 * 109 \quad \mathrm{~b}$ \\
\hline
\end{tabular}

Means in the same column followed by different letters are significantly different using the Tukey test $(\mathrm{P}<0.05)$

Table 3. Interaction between coat colour and breed in alpacas for the total volume of skin (Vref), total volume of the derma (VD) in $\mathrm{mm} 3$

\begin{tabular}{|c|c|c|c|}
\hline & & Light & Dark \\
\hline \multirow[t]{2}{*}{ Vref } & Huacaya & $4.2 * 109$ & $3.8 * 109$ \\
\hline & Suri & $4.7 * 109$ & $5.6 * 109 \mathrm{~b}$ \\
\hline \multirow[t]{2}{*}{ VD } & Huacaya & $1.3 * 109$ & $1.1 * 109 \mathrm{a}$ \\
\hline & Suri & $1.7 * 109 \mathrm{~A}$ & $2.5 * 109 \mathrm{Bb}$ \\
\hline
\end{tabular}

Means in the same column followed by different lower case letters and in the same row followed by upper case letters are significantly different using the Tukey test $(\mathrm{P}<0.05)$

Table 4. Correlations between coat traits in Alpacas

\begin{tabular}{|l|r|r|r|r|}
\hline & VRef & VGF & VD & VGSe \\
\hline VvGF & 0.72 & & & \\
\hline VvD & 0.85 & 0.31 & & \\
\hline VvGSe & 0.11 & 0.14 & 0.12 & \\
\hline VvGSu & 0.56 & 0.37 & 0.51 & 0.10 \\
\hline
\end{tabular}

Total volume of skin (Vref), total volume of the derma (VD), follicular groups (VGF), sebaceous (VGSe) and sweat glands (VGSu) 
The principal component analysis showed a strong relationship between all traits except VGSe (Fig. 6).

\section{Discussion}

In the macroscopic analysis of alpaca skin and fiber fragments, two layers of the skin were observed: the epidermis and the dermis. Inside the follicular groups containing the hair, the structure was similar to that found in other mammals. The skin is an important organ as it is a protective barrier which acts as a defense of the body to the external environment $[19,20,15,23,24,11]$. The set of hairs that emerge from the epidermis form the fleece $[16,17]$, protecting animals in extremely cold climates.

Unlike other authors, it was not possible to view all epidermis layers. The basal stratum contained cubic cells. In turn, the stratum corneum of dead keratinized cells was observed, which appeared as a detached layer from the rest of the other strata of the epidermis. However, our data are in agreement with the previous cited authors $[11,14$, $18,21,22,25,26]$ and the alpaca epidermis constitutes a very thin layer in the scapular, costal and lateral femoral regions, more precisely parts of the lateral regions of the thoracic limb, thorax and pelvic limb.

The dermis was also analyzed, which had two parts, the papillary dermis, closer to the epidermis and the reticular but deep dermis, near the hypodermis. The difference between both portions was not evident. In the dermis, we found glandular tissue composed of sebaceous glands and sweat glands. The sweat glands were visualized as tubular glands responsible for the thermal regulation and elimination of toxic substances. They produce sweat, mostly composed of water, mineral salts, some proteins and fatty substances $[11,12,15,22,23,25,27,28]$.

In the present study, we evaluated the costal, scapular and lateral femoral regions, which were associated with the hair follicles. These were composed of rounded 
cells with spherical nuclei. In other studies, where other regions were analyzed, the authors did not find these glands in the interdigital space [27].

For alpacas, this gland is extremely important because it confers softness, texture and resistance to its fibers [10-14, 21-25], which makes a difference to the analysis of fiber quality, determining the factors required and required by the textile industries [29-30].

In the reticular dermis, the hair bulbs were surrounded by dense connective tissue with an oblique disposition. These did not to emerge uniformly. Surrounding the dermal papilla were melanocytes within the hair cortex. The hair cuticle and two root sheaths were also observed.

It was possible to separate the follicular groups of hair follicles, which could be primary or secondary, being responsible for the formation of primary and secondary hair, respectively. Hair follicles were found both inside and outside the follicular groups. Authors found one to three primary follicles within a follicular group, and between 20 and 33 secondary follicles $[3,31-33]$.

Collagen is a matrix protein, that gives rigidity and consistency to the skin, making dermis structures more stable inside the dermal tissue, serving as a basis for sustaining and nourishing hair $[15,21,23,26]$. Masson's trichrome stains showed bluestained collagen fibers compatible with type I collagen fibers. These bypassed the follicular groups, offering support to them as well as the other structures in the dermis. In the case of the slides stained with Picrosirius Red and observed under polarized light, the dermis was visualized, which presented fibers of red color, indicating collagen type I fibers. In the deepest dermis, the presence of red, orange and green polarized fibers was observed, the latter being type III collagen fibers, or reticular fibers, which are small and provide a supporting the whole structure. 
Through scanning electron microscopy analysis, it was possible to evaluate the components present in the integument, as well as the microstructures, but higher resolutions would be needed to identify them. It was possible to observe the two layers of the skin separated by the basal layer. In the epidermal layer, we could visualize the stratum corneum. This layer is the last superficial layer of the epidermis responsible for the protective barrier $[11,15,19-23]$.

Through scanning electron microscopy, it was possible to see that there were follicles with only one hair or a set of fibers emerging from the same follicular group, as also described by $[11,19,20,25]$. As hairs emerge they come together to share the same follicle. This is because secondary follicles are responsible for the cover hair, which help maintain thermal regulation $[16,17]$. Therefore, they do not need to be very thick, just be numerous to make up the total body coat of the animal.

Scanning electron microscopy was used to determine the hair diameter of the costal region. This is the region considered as representative of the alpaca integument $[3,10,23,32]$. In the light and dark alpacas of the Huacaya and Suri varieties, the diameter ranged from $12.65 \mu \mathrm{m}$ to $55.00 \mu \mathrm{m}$. [34] measured fibers of various species, finding diameters varying from $18 \mu \mathrm{m}$ to $30 \mu \mathrm{m}$ for alpacas; <15 $\mu \mathrm{m}$ for vicuñas; from $15 \mu \mathrm{m}$ to $18 \mu \mathrm{m}$ for guanacos; > $20 \mu \mathrm{m}$ for llamas; from $12 \mu \mathrm{m}$ to $18 \mu \mathrm{m}$ for cashmere (double fleece goats); from $22 \mu \mathrm{m}$ to $30 \mu \mathrm{m}$ for mohair (single fleece goats); $17 \mu \mathrm{m}$ for Merino sheep; > $30 \mu \mathrm{m}$ for Scottish Blackface sheep; from $10 \mu \mathrm{m}$ to $13 \mu \mathrm{m}$ for Angora rabbits. According to Peruvian Technical Standards, for alpacas a fiber of at most $21.50 \mu \mathrm{m}$ is the most valued fine fiber for the textile industry. This is because it provides a softer and more comfortable fiber for the production of fabrics, whereas the average fiber is 26.60 $\mu \mathrm{m}$ to $29 \mu \mathrm{m}$, and the thickest fiber of $31.60 \mu \mathrm{m}$ is not used in the textile industry because they are not comfortable fibers to produce clothing. 
The presence of different matrix proteins from the three body regions of the tegument of the light and dark alpacas of the Huacaya and Suri varieties was analyzed. First, descriptions were made for the various types of collagen (I, III and IV), where the marking for type I and type IV collagen was observed throughout the epidermal and dermal tissue, together with their glandular structures, and hair follicles. In the case of collagen III, only the marking for dermis collagen fibers was observed.

The S100 protein was also observed, important for the integument, because it is present in melanocytes, cells derived from the neural crest and is also present in Langerhans cells and keratinocytes. In this study, all skin structures such as epidermis, dermis, sebaceous and sweat glands showed positive labeling for this protein. Hair follicles were more marked than in the other structures of the integument. For the three body regions of the light and dark alpacas of the Huacaya and Suri varieties, the presence of melanocytes is similar and this marker may express any of its pigment forms such as eumelanins (dark colors like brown or black) or pheomelanins (reddish or yellow colors) [34].

More follicular groups and skin volume were seen in the femoral region indicating higher fiber production in this region. In dark skinned animals, more skin volume was seen in the Suri compared to the light colored animals and Dark Suri produced more derma than light-skinned animals or Huacaya. The principal component analysis shows the close relationship between total volume of the derma (VD), follicular groups (VGF), skin volume (Vref) and sweat glands (VGSu). This indicates that for fiber production, selection for one of these traits should increase the others. This may be useful for breeders when selecting animals for reproduction in breeding schemes. 


\section{Conclusions}

Our study concluded that the femoral region presented higher fiber production. Dark animals had more derma and it was reported close relationship between total skin volume and their fractions volumes: derma, follicular groups and sweat glands.

\section{Acknowledgments}

Thanks are due to CAPES for funding and scholarships.

\section{Authors' contributions}

LMDN e MAM conceived and designed the experiment

LMDN, JB, MK, ADC, ACOV performed and analysed the immunohistochemistry

LMDN, THCS, SPG, CMM performed the stereological design and analysis

LMDN, ACOC, THCS, CMM, MAM analysed the data and wrote the paper

\section{Funding}

Coordination for the Improvement of Higher Education Personnel (CAPES) - Brazil

\section{Availability of data and materials}

All analyzed data from this study are included in this manuscript

\section{Ethics approval}

The experiment was approved by the Animal Use Ethics Committee of the Faculty of Veterinary Medicine and Animal Science of the University of São Paulo (CEUA/FMVZ) under No. 4781170317.

\section{Consent for publication}

Not applicable

\section{Competing interest}

The authors declare that they have no competing interest

\section{References}

1. Minagri. Situación de las actividades de crianza y producción. Origen y Situación 
actual. 2019. http://www.minagri.gob.pe/portal/?id=298\&limitstart=0 Accessed 19 nov 2019.

2. Gutiérrez Hermosa, L. Las normas técnicas peruanas y su impacto en el desarrollo de los camélidos peruanos.

2019.http://www.minagri.gob.pe/portal/download/pdf/herramientas/cendoc/videoconfer encias/2011/normas_tecnicas_camelidos_13dic11.pdf Accessed 19 nov 2019.

3. Antonini M, Gonzales M, Valbonesi, A. Relationship between age and postnatal skin follicular development in three types of South American domestic camelids. Livest Prod Sci. 2004; 90(2-3): 241-246.

4. Montes M, Quicaño I, Quispe R, Quispe E, Alfonso L. Quality characteristics of Huacaya alpaca fibre produced in the Peruvian Andean Plateau region of Huancavelica. Span J of Agric Res. 2008; 6 (1): 33-38.

5. Cervantes I, Goyache F, Burgos A, Pérez-Cabal MA, Morante R, Gutiérrez JP. Genetic improvement for alpaca fibre production in the Peruvian Altiplano: the Pacomarca experience. Anim Genet Resour Inf 2009; 45: 37

6. Gutiérrez JP, Goyache F, Burgos A, Cervantes I. Genetic analysis of six production traits in Peruvian alpacas. Livest Sci. 2009; 123 (2-3):193-197.

7. Oria I, Quicaño I, Quispe E, Alfonso L. Variabilidad del color de la fibra de alpaca en la zona altoandina de Huancavelica-Perú. Anim Genet Resour Inf. 2009; 45: 79, 2009.

8. Quispe EC, Rodríguez TC, Iñiguel LR, Mueller JP. Producción de fibra de alpaca, llama, vicuña y guanaco en Sudamérica. Anim Genet Resour Inf. 2009; 45: 1-14.

9. Vélez V, Salazar J, Pacheco J, Pezo D, Franco F. Histología cuantitativa de la piel de alpaca diferenciada por calidad de fibra. Arch Latinoam Pro Anim. 2016; 24: 7-10.

10. Junqueira LC, Carneiro J. Histologia Básica. 12. ed. Rio de Janeiro: Guanabara Koogan, 2013.

11. Torres de Jasaui J, Vélez V, Zegarra J, Díaz G. Caracterización de la histología de la piel de alpaca. Sitio Argent Produc Anim. 2007; 1-8.

12. Moore KE, Blache D, Maloney SK. Fibre diameter and insulation in alpacas: The biophysical implications. Small Ruminant Res. 2011; 96 (2-3):165-172.

13. Vélez V, Salazar J, Pacheco J, Pezo D, Franco F. Histología cuantitativa de la piel de alpaca diferenciada por calidad de fibra. Arch Latinoam Prod Anim. 2016; 24 (7): 710 .

14. Atlee BA, Stannard AA, Fowler ME, Willemse T, Ihrke PJ, Olivry T. The histology of normal llama skin. Vet Dermatol. 1997; 8 (3): 165-176.

15. Grigg GC, Beard LA, Augee ML. The Evolution of Endothermy and Its Diversity in Mammals and Birds. Physiol Biochem Zool. 2004; 77 (6): 982-997. 
16. Gerken M. Relationships between integumental characteristics and thermoregulation in South American camelids. Animal. 2010; 4 (9): 1451-1459.

17. Tolosa EMC, Rodrigues CJ, Behmer AO, Neto AGF. Manual de Técnicas para Histologia Normal e Patológica. 2nd ed. São Paulo: Manole; 2003.

18. Getty R. Anatomia dos Animais Domésticos. 5th ed. Rio de Janeiro: Guanabara Koogan; 1986.

19. Gundersen HJG, Jensen EBV, Kieu K, Jensen J. The efficiency of systematic sampling in stereology—reconsidered. J Microsc.1999; 193:199-211.

20. Howard CV, MG Reed. Unbiased Stereology. Three-Dimensional Measurement in Microscopy. 2nd ed. Liverpool: QTP Publications; 2010.

21. Banks W J. Histologia Veterinária Aplicada. 2nd ed. São Paulo: Manole; 1991.

22. Samuelson DA. Tratado de Histologia Veterinária. 1st ed. Rio de Janeiro: Saunders Elsevier; 2007.

23. Vélez V, Torres J, Leyva V, García, W. Histología Descriptiva De La Piel De Alpaca. ublicado en el V CONGRESO MUNDIAL SOBRE CAMÉLIDOS 17 al 21 de noviembre 2009 Riobamba - Chimborazo - Ecuador. 2009; 9.

24. Lacolla D, García M, Corredera C, Buey V. Estructura histológica de la piel de los camélidos sudamericanos. Cient vet. 2010; 12 (1): 8-15.

25. Bustinza V. La alpaca: conocimiento del gran potencial andino. 2001. Puno, Perú: Oficina de Recursos del Aprendizaje - Sección Publicaciones UNA - Universidad Nacional del Altiplano.

26. Fleis RI, Scott DW. The Microanatomy of Healthy Skin from Alpacas (Vicugna Pacos). J Vet Diagn Investig. 2010; 22: 716-719.

27. Meruane M, Rojas M. Desarrollo de la Piel y sus Anexos en Vertebrados Skin and Appendages Development in Vertebrates. Int. J. Morphol. 2012; 30 (4):1422-1433, 2012.

28. Wang X, Wang L, Liu X. The Quality and Processing Performance of Alpaca Fibres A report for the Rural Industries Research and Development Corporation. 2003; 03.

29. Peña Q, Gutiérrez P, Unanua P. Caracteristicas Productivas Y Textiles De La Fibra De Alpacas De Raza Huacaya. Revista Complutense De Ciencias Veterinarias. 2013; 7 (1):1-29.

30. Mucha A, Janeczek M. Morphological and elemental analysis of alpaca hair (Vicugna pacos) using scanning electron microscopy with energy dispersive X-ray spectroscopy (SEM - EDX). Med Weter. 2018; 74 (1): 6046-2018. 
31. Badajoz E, Sandoval N, Garcia W, Pezo D. Descripción histológica del complejo folicular piloso en crías de alpacas. Revista de Investigaciones Veterinarias. 2009; 20, (2):154-164.

32. Antonini, M. Hair follicle characteristics and fibre production in South American camelids. Animal. 2010; 4 (9): 1460-1471.

33. Molina G, Teich I, Antonini M, Renieri C, La Terza A, Balzarini M. Spatial structure of skin follicles in Suri and Huacaya alpacas. Small Ruminant Res. 2016; 140: $22-26$.

34. Galbraith, H. Fundamental hair follicle biology and fine fibre production in animals. Animal. 2010; 4 (9): 1490-1509.

\section{Legend of figures}

Fig 1. Scanning electron micrograph. Sections to the skin of the Huacaya and Suri alpacas in A show the hair (p), the dermis (d) and the hypodermis (h). Follicular groups (gf) (B). In $\mathrm{C}$, in greater increase, the follicular groups (gf). In D, the hair with the cuticle (arrow) is observed, also in E, the epidermis (e) and the dermis (d), separated by the basal layer of the epidermis (arrow). In F, the fibers of a follicular group (gf) emerging from the hair follicle in the papillary dermis. In G, follicular group fibers (gf) and sebaceous glands (gse) (arrow). Skin longitudinal sections, the follicular groups in the dermis (gf) (H), in I, greater increase of the follicular groups (gf). In J, sweat gland (gsu) indicated by the arrow between two follicular groups (gf). In K, follicular group (gf) composed of four hair follicles, accompanied by the sebaceous gland (gse). In L, the follicular group (gf) on the side is observed the primary follicle (fp). In M, primary follicle (fp) containing medullary primary hair (p) with visible cuticle in the form of layers (arrow). In N, the primary follicle (fp) with the medullary primary hair (p). In $\mathrm{O}$, the hair follicle layers and the hair (p).

Fig 2. Photomicrograph of the skin. In $A$ and $B$ the dermis (d) and hypodermis (h). In $C$ and $\mathrm{D}$, the epidermis (e) and the dermis (d), the junction between the epidermis and the dermis (arrow). In E, follicular groups (gf), and sebaceous gland (gse). In F, longitudinal section to the skin with follicular groups (gf). In G, follicular group (gf) with sweat gland (gsu) inside. In H, hair follicle (fp) containing fibers (f) inside. In I, hair follicle containing a fiber (f) with marrow (*). In J, K and L, hair bulb (b). Staining: Hematoxylin eosin. 
Fig 3. Photomicrograph of the skin. The structures stained in blue represent the collagen fibers. In A and B, skin cross sections; epidermis (e), dermis (d) and junction of epidermis with dermis (arrow). In C hair follicle (fp). In D, dermis (d) and follicular groups (gf). In E, follicular groups (gf) with sebaceous glands (arrows) and within the circle the sweat gland, just as in F. In G and H, hair bulb (b). Skin longitudinal sections; follicular groups (gf), hair follicle with marrow (*), in I-L. Staining: Masson thricrome.

Fig 4. Photomicrograph of the skin cross sections (A-F). In A, dermis (d), follicular groups (gf) and hypodermis (h). In B, the arrow indicating the separation between the epidermis (e) and the dermis (d). In C, follicular groups (gf) and hypodermis (h). In D, follicular groups (gf), collagen fibers are observed in red for dermis (d) and hypodermis (h). E, hair follicles (fp), the dermis (d) with red collagen fibers and the arrow indicating division with the epidermis (e). In F, follicular groups, surrounded by red and orange collagen fibers, hypodermis (h). Longitudinal cuts to the skin (G-L). In G, follicular group (gf), containing sebaceous glands indicated by arrows. In H, follicular groups (gf) with sweat glands (gsu). In I, sweat glands within the circle. In J, follicular group (gf) surrounded by collagen fibers, in the arrows sebaceous glands, which were not observed in polarized light. In K, dermal collagen fibers in red and orange surrounding the follicular groups (gf) and sweat glands (gsu), also in L, follicular groups (gf) and sebaceous glands within the circle. Staining: Picrossirius red.

Fig 5. Lateral femoral skin longitudinal sections immunostained collagen I (A-D) Collagen III (E-H), Collagen IV (I-M), S100 (N-Q), Control (R-U). In the images the follicular groups (gf) are observed in light-fiber Huacaya (A, E, I, N, R), dark-fiber Huacaya (B, F, J, O, S), light-fiber Suri (C, G, L, P, T), dark-fiber Suri (D, H, M, Q, U).

Fig 6. First two principal components for coat traits in alpacas. Total volume of skin (Vref), total volume of the derma (VD), follicular groups (VGF), sebaceous (VGSe) and sweat glands (VGSu). 


\section{Figures}
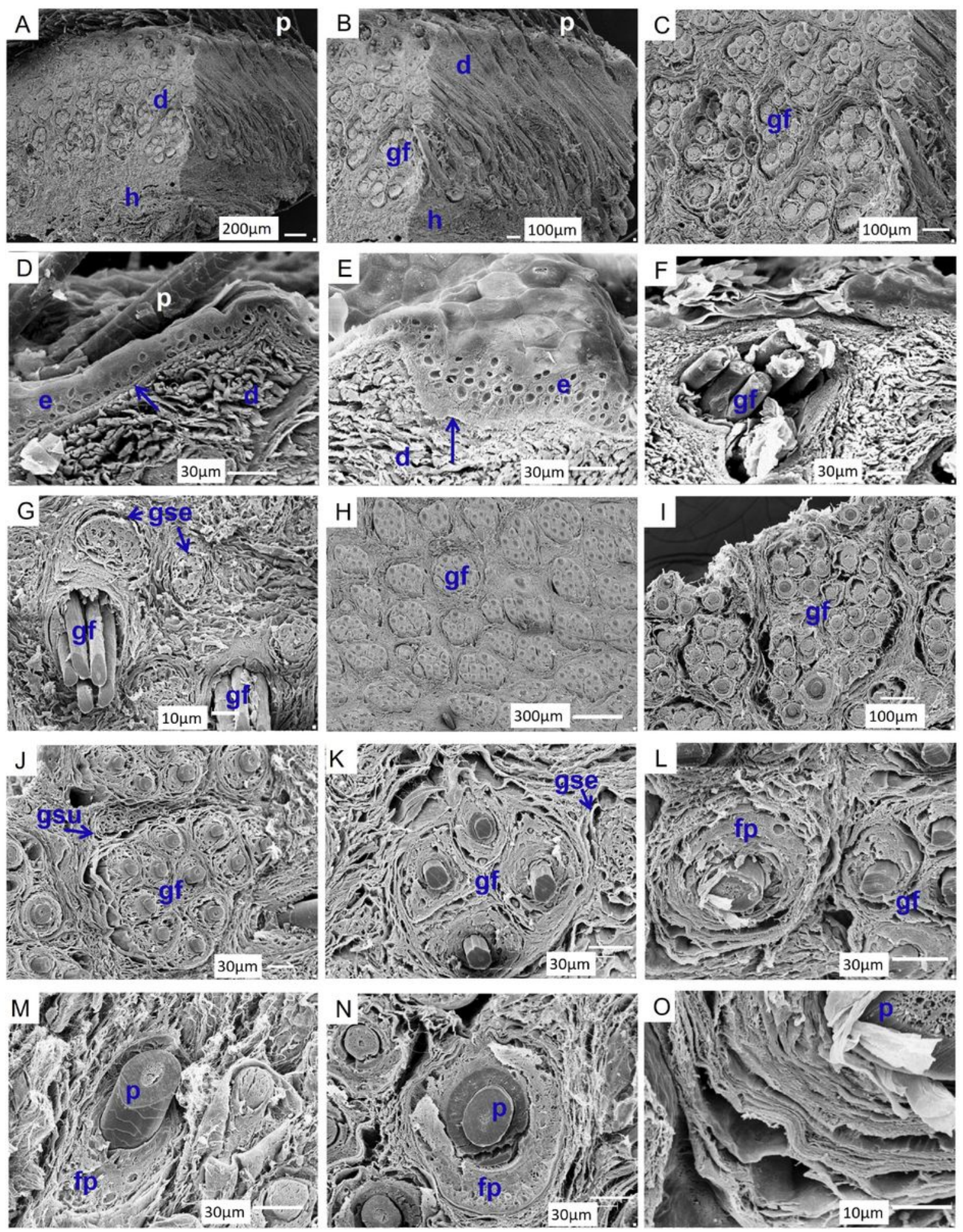

\section{Figure 1}

Scanning electron micrograph. Sections to the skin of the Huacaya and Suri alpacas in A show the hair (p), the dermis (d) and the hypodermis (h). Follicular groups (gf) (B). In C, in greater increase, the follicular groups (gf). In D, the hair with the cuticle (arrow) is observed, also in $E$, the epidermis (e) and the dermis 
(d), separated by the basal layer of the epidermis (arrow). In F, the fibers of a follicular group (gf) emerging from the hair follicle in the papillary dermis. In G, follicular group fibers (gf) and sebaceous glands (gse) (arrow). Skin longitudinal sections, the follicular groups in the dermis ( $g f)(H)$, in I, greater increase of the follicular groups (gf). In J, sweat gland (gsu) indicated by the arrow between two follicular groups (gf). In K, follicular group (gf) composed of four hair follicles, accompanied by the sebaceous gland (gse). In L, the follicular group (gf) on the side is observed the primary follicle (fp). In M, primary follicle (fp) containing medullary primary hair $(\mathrm{p})$ with visible cuticle in the form of layers (arrow). In N, the primary follicle $(f p)$ with the medullary primary hair $(p)$. In 0 , the hair follicle layers and the hair $(p)$. 

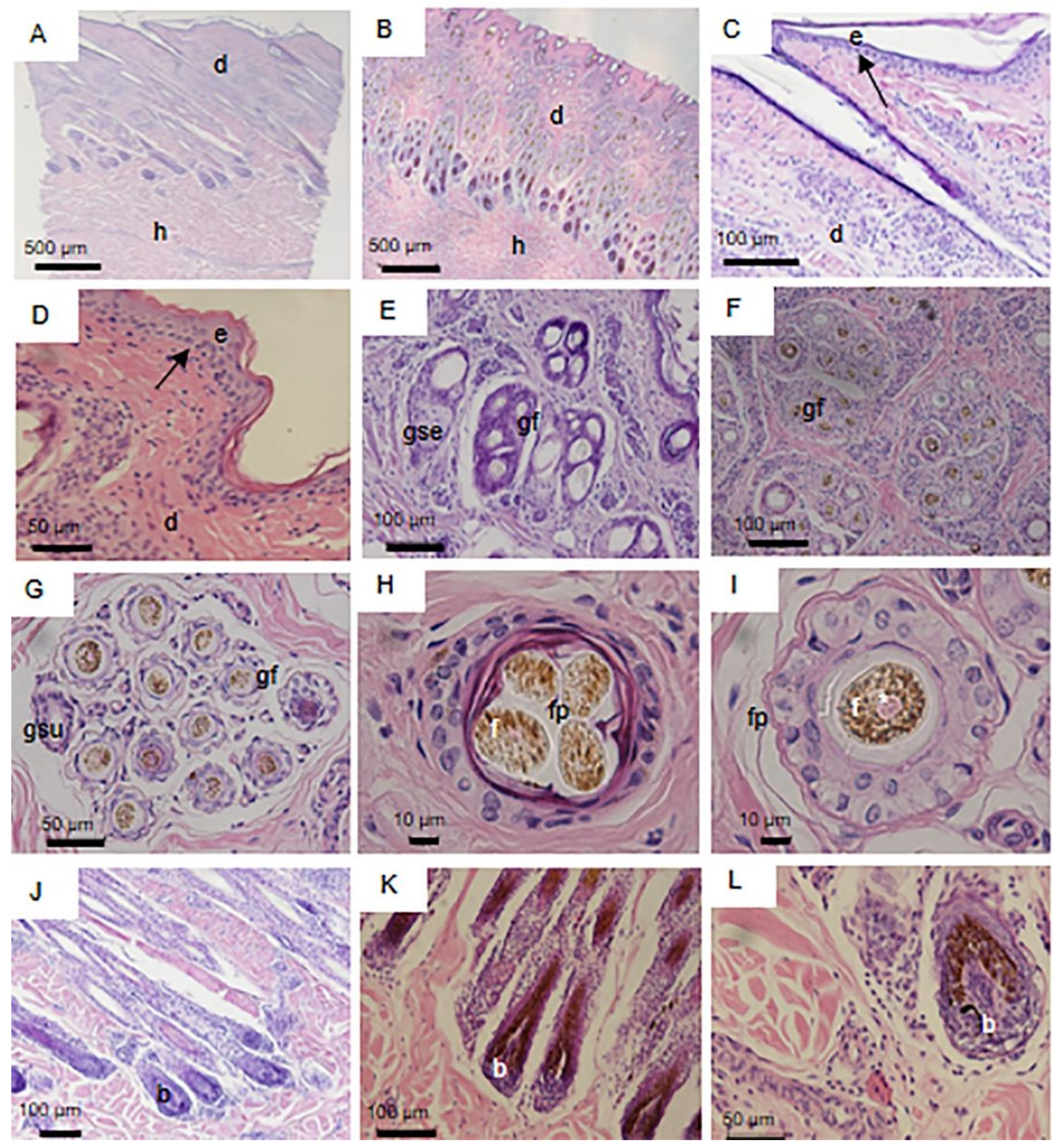

\section{Figure 2}

Photomicrograph of the skin. In A and B the dermis (d) and hypodermis ( $h$ ). In C and D, the epidermis (e) and the dermis (d), the junction between the epidermis and the dermis (arrow). In E, follicular groups (gf), and sebaceous gland (gse). In F, longitudinal section to the skin with follicular groups (gf). In G, follicular group (gf) with sweat gland (gsu) inside. In $\mathrm{H}$, hair follicle (fp) containing fibers (f) inside. In I, hair follicle containing a fiber (f) with marrow $\left({ }^{*}\right)$. In J, K and L, hair bulb (b). Staining: Hematoxylin eosin. 

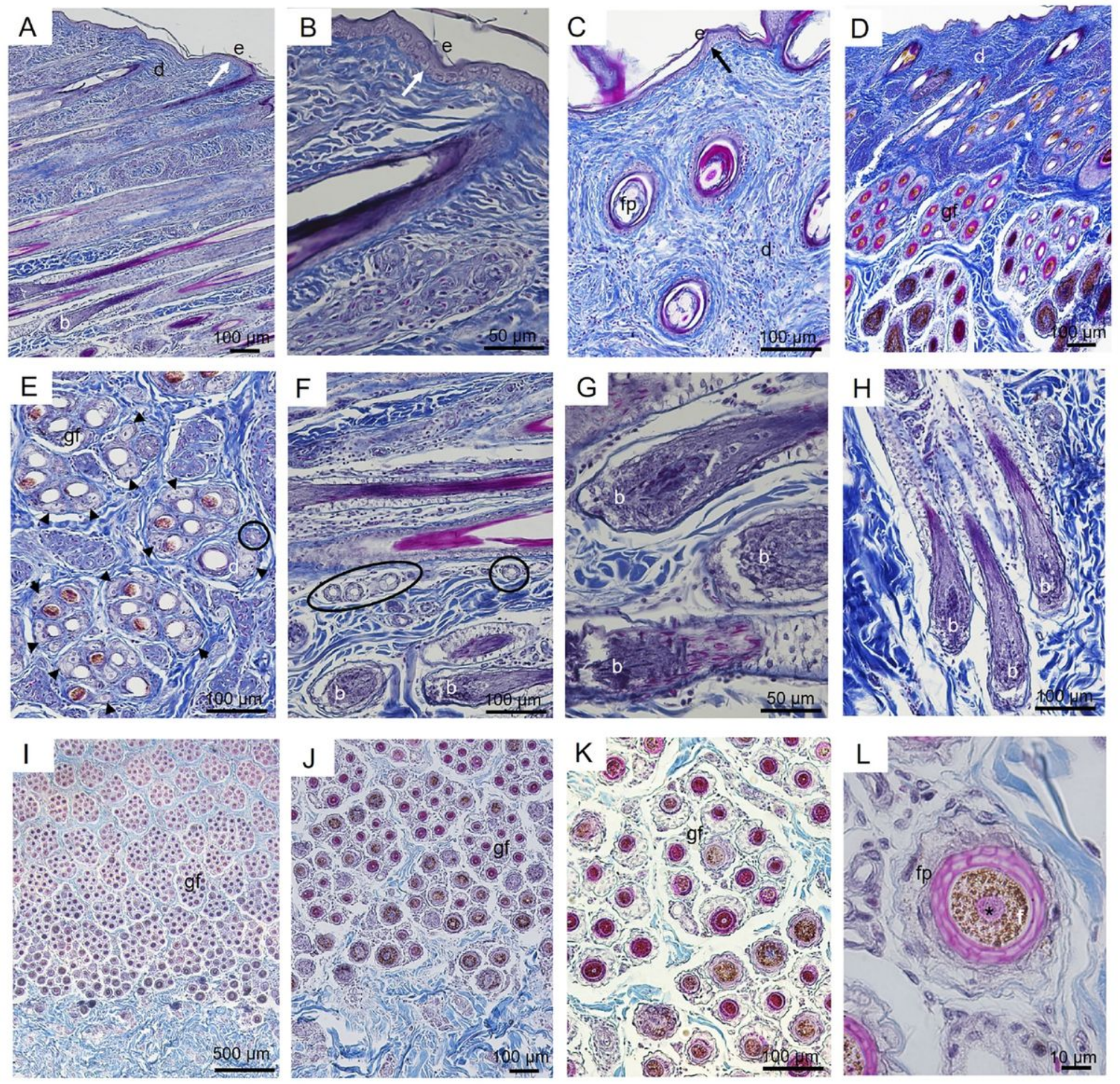

\section{Figure 3}

Photomicrograph of the skin. The structures stained in blue represent the collagen fibers. In A and B, skin cross sections; epidermis (e), dermis (d) and junction of epidermis with dermis (arrow). In $\mathrm{C}$ hair follicle (fp). In D, dermis (d) and follicular groups ( $g f)$. In E, follicular groups (gf) with sebaceous glands (arrows) and within the circle the sweat gland, just as in $\mathrm{F}$. In $\mathrm{G}$ and $\mathrm{H}$, hair bulb (b). Skin longitudinal sections; follicular groups (gf), hair follicle with marrow $\left({ }^{*}\right)$, in I-L. Staining: Masson thricrome. 

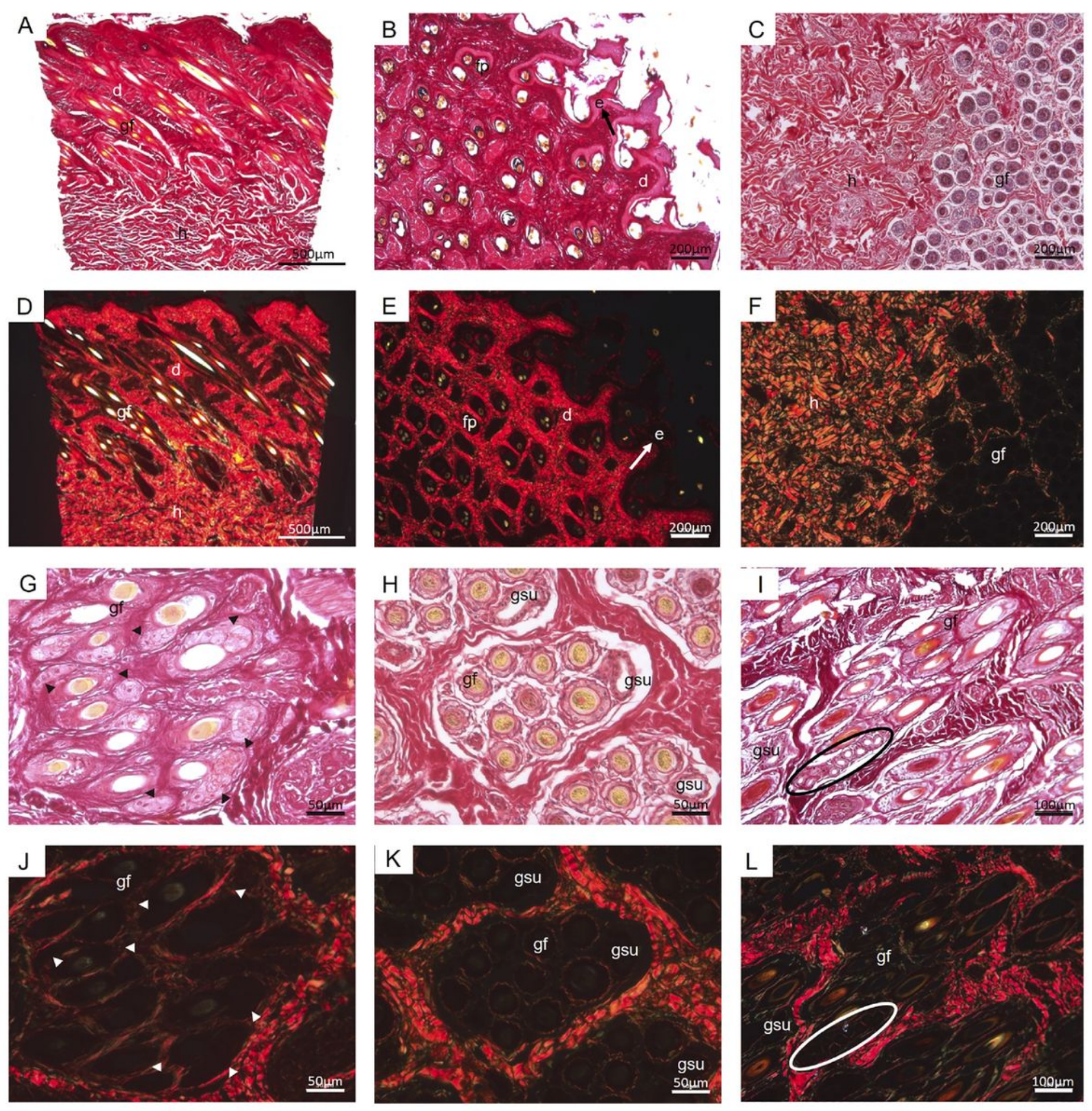

\section{Figure 4}

Photomicrograph of the skin cross sections (A-F). In A, dermis (d), follicular groups (gf) and hypodermis (h). In B, the arrow indicating the separation between the epidermis (e) and the dermis (d). In C, follicular groups (gf) and hypodermis (h). In D, follicular groups (gf), collagen fibers are observed in red for dermis (d) and hypodermis (h). E, hair follicles ( $f p)$, the dermis (d) with red collagen fibers and the arrow indicating division with the epidermis (e). In F, follicular groups, surrounded by red and orange collagen fibers, hypodermis (h). Longitudinal cuts to the skin (G-L). In G, follicular group (gf), containing sebaceous 
glands indicated by arrows. In $\mathrm{H}$, follicular groups (gf) with sweat glands (gsu). In I, sweat glands within the circle. In J, follicular group ( $\mathrm{gf}$ ) surrounded by collagen fibers, in the arrows sebaceous glands, which were not observed in polarized light. In $\mathrm{K}$, dermal collagen fibers in red and orange surrounding the follicular groups (gf) and sweat glands (gsu), also in L, follicular groups (gf) and sebaceous glands within the circle. Staining: Picrossirius red.

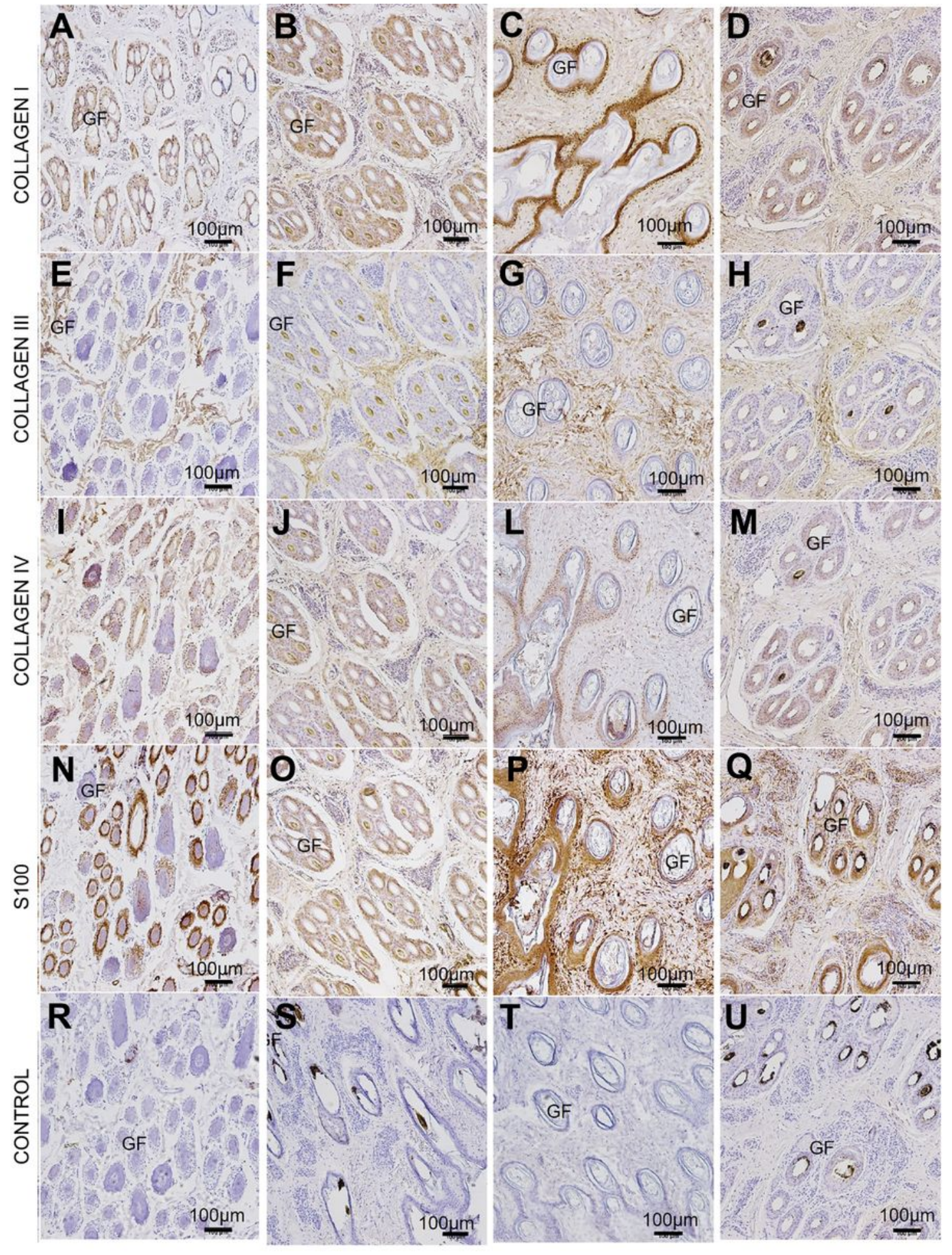

Figure 5 
Lateral femoral skin longitudinal sections immunostained collagen I (A-D) Collagen III (E-H), Collagen IV (I-M), S100 (N-Q), Control (R-U). In the images the follicular groups (gf) are observed in light-fiber Huacaya $(A, E, I, N, R)$, dark-fiber Huacaya $(B, F, J, O, S)$, light-fiber Suri $(C, G, L, P, T)$, dark-fiber Suri $(D, H, M, Q, U)$.

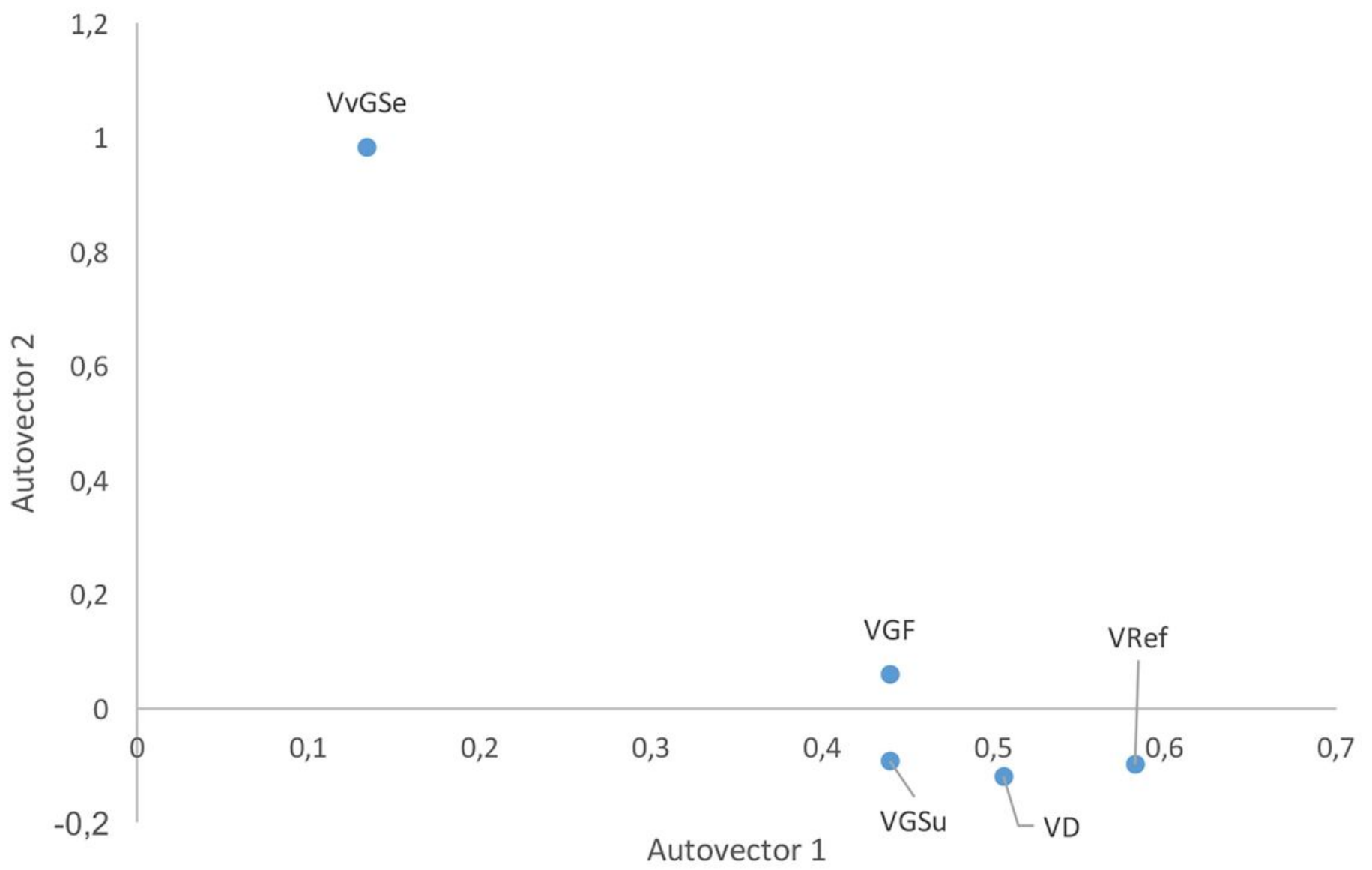

Figure 6

First two principal components for coat traits in alpacas. Total volume of skin (Vref), total volume of the derma (VD), follicular groups (VGF), sebaceous (VGSe) and sweat glands (VGSu). 